\title{
Editorial: Multiple Stressors Across Ecosystem Boundaries
}

\author{
Ellard R. Hunting ${ }^{1,2 \star}$, Mirco Bundschuh ${ }^{3,4}$ and Maarten Schrama ${ }^{5}$ \\ 'School of Biological Sciences, University of Bristol, Bristol, United Kingdom, ${ }^{2}$ Department of Biology, Woods Hole \\ Oceanographic Institution, Woods Hole, MA, United States, ${ }^{3}$ Institute for Environmental Sciences, University of \\ Koblenz-Landau, Landau, Germany, ${ }^{4}$ Department of Aquatic Sciences and Assessment, Swedish University of Agricultural \\ Sciences, Uppsala, Sweden, ${ }^{5}$ Institute of Environmental Sciences, Leiden University, Leiden, Netherlands
}

Keywords: pesticides, artificial light, climate change, nutrients, atmospheric electricity, food webs, bioturbation

\section{Editorial on the Research Topic}

\section{Multiple Stressors Across Ecosystem Boundaries}

OPEN ACCESS

Edited by:

Oladele Ogunseitan University of California, Irvine, United States

Reviewed by:

Frontiers Editorial Office,

Frontiers Media SA, Switzerland

*Correspondence:

Ellard R. Hunting

e.r.hunting@bristol.ac.uk

Specialty section:

This article was submitted to

Environmental Toxicology,

a section of the journal

Frontiers in Environmental Science

Received: 16 May 2019

Accepted: 12 June 2019

Published: 03 July 2019

Citation:

Hunting ER, Bundschuh M and Schrama M (2019) Editorial: Multiple

Stressors Across Ecosystem Boundaries. Front. Environ. Sci. 7:98.

doi: 10.3389/fenvs.2019.00098
While the natural environment already sets harsh conditions for organisms, human activities can add additional constraints to organisms and the ecosystem processes they sustain. Increasing our understanding of human influences is therefore essential to facilitate reliable and effective legislative intervention. However, attaining predictions on how stressors affect the environment is notoriously challenging and to date failed to tackle ongoing declines in biodiversity (Johnson et al., 2017). Historically, scientific studies have mainly focused on single anthropogenic stressors and ecosystems compartments (e.g., European Food Safety Authority Panel on Plant Protection Products and Their Residues, 2013: Guidelines for risk assessment). This approach limits our ability to generalize and predict ecosystem responses, as it is increasingly recognized that multiple anthropogenic and natural stressors can interact and resonate beyond the boundaries of single ecosystems (e.g., Schulz et al., 2015; Gessner and Tlili, 2016; Vijver et al., 2017; Graf et al., 2019; Ogunseitan, 2019). Therefore, it is necessary to unite research that addresses the combined impact of stressors or its propagation across ecosystem boundaries.

The majority of ecosystems is contaminated with multiple chemical pollutants (Schreiner et al., 2016). These chemicals interact to alter food webs by directly affecting organisms or indirectly by altering the available resources (e.g., O'Gorman et al., 2012; Hunting et al., 2016; Zhai et al., 2018). Schrama et al. support this idea by highlighting that nutrients, a herbicide and an insecticide jointly modify trophic linkages within a model aquatic food web. More specifically, the results suggest that autotrophic parts of the food web are mainly controlled by single and joint effects of nutrients and herbicides, while the heterotrophic part of the food web is impacted by nutrients and insecticides as well as their interplay. It remains a challenge to determine how these interactions in a simple food web translate to complex environments, including soils, marine sediments, and freshwater sediments. This lack of a proper understanding of the effects of stressors may partly stem from system-specific interactions between stressors and biota, which may overshadow the directly generalizable effect of stressors. For example, Van der Meer et al. show that, the higher the activity of sediment-dwelling invertebrates, the more sensitive they become to sediment associated contaminants. This suggests that biotic interactions with their chemical environment reinforce the effect of stressors by promoting the release of contaminants across the sediment-water interface. 
A long-standing question has been to determine how to investigate the impact of multiple stressors on biotic interactions in a spatio-temporally heterogeneous environment. Franken et al. rejuvenate a widely adopted approach to partition the impact of a range of stressors on natural soil communities which allows them to assess the relative importance of extreme climate events in a complex environment. Similarly, a combination of biotic (soil compaction by livestock grazing; plant stress resistance) and abiotic (salinity, hypoxia) stressors were observed to largely explain complex patterns in vegetation cover across the marineterrestrial interface (Veldhuis et al.). Despite the complexity associated with disentangling the effects of multiple stressors in complex environments, these studies hint that valuable information can be obtained from a mix of complementary approaches, including lab and mesocosm experiments as well as field observations. To further this research field on the combined impact of stressors across ecosystem continuums, Bruder et al. provide a novel perspective to include food-web characteristics and biotic interactions in analyses of multiple-stressor effects on ecosystems. The strength of their approach is that it can be used on biomonitoring data as well as experimental data, and, by providing an integrated approach to study biotic interactions in a multiple stressor context, can help us generalize and predict ecosystem responses.

The atmosphere is also an important, yet frequently overlooked compartment that facilitates the propagation of stressors through multiple ecosystems. For instance, plastic particles are transferred from aquatic systems via the atmosphere to the terrestrial food web, through the emergence of aquatic mosquito-larvae (Al-Jaibachi et al., 2018). Another profound example of how humans alter their environment is artificial light (e.g., street lights). Manfrin et al. convincingly show in this research topic that artificial light can cause shifts in the abundance of night- and day-active insects, and lead to an increased aquatic-to-terrestrial subsidy flux. This illustrates that alterations induced by human activity-here the physical environment-can have major repercussions for functioning of networks of connected ecosystems. On a larger scale,

\section{REFERENCES}

Al-Jaibachi, R., Cuthbert, R. N., and Callaghan, A. (2018). Up and away: ontogenic transference as a pathway for aerial dispersal of microplastics. Biol. Lett. 14:20180479. doi: 10.1098/rsbl.2018.0479

Engels, S., Schneider, N. L., Lefeldt, N., Hein, C. M., Zapka, M., Michalik, A., et al. (2014). Anthropogenic electromagnetic noise disrupts magnetic compass orientation in a migratory bird. Nature 509, 353-356. doi: 10.1038/nature 13290

European Food Safety Authority Panel on Plant Protection Products and Their Residues (2013). Guidance on tiered risk assessment for plant protection products for aquatic organisms in edge-of-field surface waters. EFSA J. 11, 3290-3558. doi: 10.2903/j.efsa.2013.3290

Gessner, M. O., and Tlili, A. (2016). Fostering integration of freshwater ecology with ecotoxicology. Freshw. Biol. 61, 1991-2001. doi: 10.1111/fwb.12852

Graf, N., Battes, K. P., Cimpean, M., Dittrich, P., Entling, M. H., Link, M., et al. (2019). Do agricultural pesticides in streams influence riparian spiders? Sci. Total Environ. 660, 126-135. doi: 10.1016/j.scitotenv.2018.12.370 the atmosphere provides a vast physical environment that connects ecosystems worldwide, and represents a complex and dynamic environment where several phenomena are known to be important for some organisms. For instance, atmospheric electromagnetic and electric fields can be used by aerial as well as terrestrial organisms (e.g., birds, spiders) for navigation and dispersal (Engels et al., 2014; Morley and Robert, 2018). However, whether the dynamic nature of atmospheric electrical phenomena is tied to belowground terrestrial and aquatic environments to date remained uncharted territory. Hunting et al. provides the first causal evidence of a link between variations in atmospheric electricity (e.g., ions and electric fields), Earth surface electrochemistry and bacterial activity in soils and sediments. The observed link is likely relevant for a wide range of organisms and prone to observed local and global changes in atmospheric electricity caused my human activities, and thereby adds a new dimension to ecosystem connectivity and the propagation of stressors.

Collectively, the work presented in this research topic raises the curtain on the effect of multiple stressors across ecosystem boundaries, and illustrates the complexity of this research field. While many examples show the consequences of stress-induced alterations in local communities at multiple organizational scales (e.g., effects across trophic levels such as prey-predator interactions), it becomes progressively more important to consider wider spatial and temporal scales and alternative propagation routes through ecosystems, seasons as well as generations (e.g., adaptation and evolution). The contributions here provide a glimpse into the breadth of work that has been done, and provides exciting and promising new research avenues that will continue to enhance our understanding of the impact of human activities along the ecosystem continuum.

\section{AUTHOR CONTRIBUTIONS}

EH drafted the manuscript. MB and MS contributed to revisions and approved the final version of the manuscript.

Hunting, E. R., Vonk, J. A., Musters, C. J. M., Kraak, M. H., and Vijver, M. G. (2016). Effects of agricultural practices on organic matter degradation in ditches. Sci. Rep. 6:21474. doi: 10.1038/srep21474

Johnson, C. N., Balmford, A., Brook, B. W., Buettel, J. C., Galetti, M., Guangchun, L., et al. (2017). Biodiversity losses and conservation responses in the Anthropocene. Science 356, 270-275. doi: 10.1126/science.aam9317

Morley, E. L., and Robert, D. (2018). Electric fields elicit ballooning in spiders. Curr. Biol. 28, 2324-2330. doi: 10.1016/j.cub.2018. 05.057

O'Gorman, E. J., Fitch, J. E., and Crowe, T. P. (2012). Multiple anthropogenic stressors and the structural properties of food webs. Ecology 93, 441-448. doi: 10.1890/11-0982.1

Ogunseitan, O. A. (2019). Embracing global warmth and climate resilience through green chemistry legislation. Hastings Environ. Law J. 25, 301-318.

Schreiner, V. C., Szöcs, E., Bhowmik, A. K., Vijver, M. G., and Schäfer, R. B. (2016). Pesticide mixtures in streams of several European countries and the USA. Sci. Total Environ. 573, 680-689. doi: 10.1016/j.scitotenv.2016. 08.163 
Schulz, R., Bundschuh, M., Gergs, R., Brühl, C. A., Diehl, D., Entling, M., et al. (2015). Review on environmental alterations propagating from aquatic to terrestrial ecosystems. Sci. Total Environ. 538, 246-261. doi: 10.1016/j.scitotenv.2015.08.038

Vijver, M. G., Hunting, E. R., Nederstigt, T. A., Tamis, W. L., van den Brink, P. J., and van Bodegom, P. M. (2017). Postregistration monitoring of pesticides is urgently required to protect ecosystems. Environ. Toxicol. Chem. 36, 860-865. doi: $10.1002 /$ etc. 3721

Zhai, Y., Brun, N. R., Bundschuh, M., Schrama, M., Hin, E., Vijver, M. G., et al. (2018). Microbially-mediated indirect effects of silver nanoparticles on aquatic invertebrates. Aquat. Sci. 80:44. doi: 10.1007/s00027-018-0594-z
Conflict of Interest Statement: The authors declare that the research was conducted in the absence of any commercial or financial relationships that could be construed as a potential conflict of interest.

Copyright (C) 2019 Hunting, Bundschuh and Schrama. This is an open-access article distributed under the terms of the Creative Commons Attribution License (CC BY). The use, distribution or reproduction in other forums is permitted, provided the original author(s) and the copyright owner(s) are credited and that the original publication in this journal is cited, in accordance with accepted academic practice. No use, distribution or reproduction is permitted which does not comply with these terms. 